PROCEEDINGS OF THE

AMERICAN MATHEMATICAL SOCIETY

Volume 140, Number 6, June 2012, Pages 1987-1995

S 0002-9939(2011)11067-0

Article electronically published on September 29, 2011

\title{
THE RIGIDITY OF DOLBEAULT-TYPE OPERATORS AND SYMPLECTIC CIRCLE ACTIONS
}

\author{
PING LI
}

(Communicated by Daniel Ruberman)

\begin{abstract}
Following the idea of Lusztig, Atiyah-Hirzebruch and Kosniowski, we note that the Dolbeault-type operators on compact, almost-complex manifolds are rigid. When the circle action has isolated fixed points, this rigidity result will produce many identities concerning the weights on the fixed points. In particular, it gives a criterion to determine whether or not a symplectic circle action with isolated fixed points is Hamiltonian. As applications, we simplify the proofs of some known results related to symplectic circle actions, due to Godinho, Tolman-Weitsman and Pelayo-Tolman, and generalize some of them to more general cases.
\end{abstract}

\section{INTRODUCTION}

This paper is concerned with the rigidity of Dolbeault-type operators on compact, almost-complex manifolds and its relationship to symplectic circle actions.

Let $\left(M^{2 n}, \omega\right)$ be a compact, connected symplectic manifold of dimension $2 n$. A circle action is called symplectic if it preserves the symplectic form $\omega$ or, equivalently, if the one form $\omega(X, \cdot)$ is closed, where $X$ is the generating vector field of this circle action. This symplectic circle action is called Hamiltonian if $\omega(X, \cdot)$ is exact; i.e., $\omega(X, \cdot)=d f$ for some smooth function $f$ on $M$. We call $f$ the moment map of this action, which is unique up to a constant.

An obvious necessary condition for a symplectic circle action to be Hamiltonian is to have nonempty fixed points corresponding to the critical points of $f$ (the minimum and the maximum of $f$ must be fixed points). In the case of Kähler manifolds [5] and of four-dimensional symplectic manifolds [17, this condition is also sufficient. However, this is not true for general higher-dimensional symplectic manifolds. In fact McDuff [17] constructed a six-dimensional symplectic manifold with a symplectic circle action which has fixed points, but which is not Hamiltonian.

Note that the fixed point sets of the counterexamples in [17 are tori. Hence one possible conjecture is that a symplectic action with isolated fixed points must

Received by the editors October 1, 2010 and, in revised form, February 2, 2011.

2010 Mathematics Subject Classification. Primary 37B05, 58J20, 32Q60, 37J10.

Key words and phrases. Rigidity, Dolbeault-type operator, symplectic circle action, Hamiltonian circle action.

The author's research is supported by the Natural Science Foundation of China (grant No. 11101308) and the Program for Young Excellent Talents in Tongji University.

(C)2011 American Mathematical Society 
be Hamiltonian. Some partially affirmative results have been obtained: TolmanWeitsman [19] showed that this conjecture holds for semifree circle actions (an action is called semifree if it is free outside the fixed points); Godinho [6] showed that this holds on certain circle actions on six-dimensional symplectic manifolds with specified weights on the fixed points.

McDuff [17] showed that a symplectic circle action is Hamiltonian if and only if there exists a connected component of the fixed point set such that all the weights of the representation of the circle action on the normal bundle of the component are positive. Therefore in order to show that a symplectic circle action is actually Hamiltonian, it suffices to show that there exists a connected component of the fixed point set satisfying this condition. The main ideas of the proof in [19] and [6] are both based on this argument. In the isolated fixed points case, Fel'dman [4] refined McDuff's observation to show that the Todd genus of a manifold admitting a symplectic circle action with isolated fixed points is either 0 , in which case the action is non-Hamiltonian, or 1, in which case the action is Hamiltonian.

In a recent paper of Pelayo and Tolman [18, the authors showed that if a compact symplectic manifold admits a symplectic (not necessarily Hamiltonian) circle action with isolated fixed points, then the weights on the fixed points must satisfy some restrictions (18, Theorem 2). We would like to point out that Theorems 1 and 3 in 18 are closely related to some much earlier work of Kosniowski (12, [13]). Theorem 1 in 18 is related to a conjecture of Kosniowski ([13, p. 338, Conjecture A). Theorem 3 in [18] has been obtained in ([12, Theorem 2) for complex manifolds and in ([13, p. 337) in the more general case. The forms of the weights on the two fixed points are hidden in the last paragraph of [12.

Our paper is inspired by the above-mentioned results. This paper is organized as follows. In Section 2, we consider some Dolbeault-type elliptic operators on compact, almost-complex manifolds. If an almost-complex manifold admits a circle action compatible with the almost-complex structure, we can define the equivariant indices of these operators under this circle action. Then following an idea of Lusztig, Atiyah-Hirzebruch and Kosniowski, we will prove the invariance of the relevant equivariant indices of these Dolbeault-type operators under circle actions having isolated fixed points. This is the meaning of the word rigidity in our title. When an almost-complex manifold admits a compatible circle action with isolated fixed points, this rigidity result immediately produces many identities concerning the weights on the fixed points. In particular, combining with Fel'dman's result 4, we give a criterion to determine whether or not a symplectic circle action is Hamiltonian.

In Section 3, as the first application of our result, we give a simple and unified new proof of Godinho's result [6], of which the original proof is very complicated (see the whole Section 3 of [6]). In fact our conclusion is more general than that of Godinho. As the second main application, we also generalize Pelayo and Tolman's above-mentioned result to circle actions on almost-complex manifolds.

Remark 1.1. Given an elliptic operator, we say it is rigid if, under any circle action, the corresponding equivariant index of this operator is invariant. A survey of the results concerning the rigidity of some important elliptic operators can be found in Section 1 of 3 . 


\section{The Rigidity of Dolbeault-Type operators AND MAIN RESUlts}

Let $\left(M^{2 n}, J\right)$ be a compact, almost-complex manifold of real dimension $2 n$ with an almost-complex structure $J$. The choice of an almost Hermitian metric on $M$ enables us to define the Hodge star operator $*$ and the formal adjoint $\bar{\partial}^{*}=-* \bar{\partial} *$ of the $\bar{\partial}$-operator ( 7$],$ p. 80$)$. Then for each $0 \leq p \leq n$, there is an elliptic differential operator (cf. [15], p. 258, Example 13.14)

$$
\bigoplus_{q \text { even }} \Omega^{p, q}(M) \stackrel{\bar{\partial}+\bar{\partial}^{*}}{\longrightarrow} \bigoplus_{q \text { odd }} \Omega^{p, q}(M)
$$

where $\Omega^{p, q}(M):=\Gamma\left(\Lambda^{p} T^{*} M \otimes \Lambda^{q} \overline{T^{*} M}\right)$. Here $T^{*} M$ is the dual of the holomorphic tangent bundle $T M$ in the sense of $J$. The index of this operator is denoted by $\chi^{p}(M)\left(=\operatorname{dim}_{\mathbb{C}} \operatorname{ker}\left(\bar{\partial}+\bar{\partial}^{*}\right)-\operatorname{dim}_{\mathbb{C}} \operatorname{coker}\left(\bar{\partial}+\bar{\partial}^{*}\right)\right)$ in the notation of Hirzebruch $[9]$. We define the Hirzebruch $\chi_{y}$-genus $\chi_{y}(M)$ by

$$
\chi_{y}(M)=\sum_{p=0}^{n} \chi^{p}(M) \cdot y^{p} .
$$

Remark 2.1. (1) When $J$ is integrable, i.e., $M$ is an $n$-dimensional complex manifold, $\chi^{p}(M)$ equals the index of the following well-known Dolbeault complex

$$
0 \rightarrow \Omega^{p, 0}(M) \stackrel{\bar{\partial}}{\rightarrow} \Omega^{p, 1}(M) \stackrel{\bar{\partial}}{\rightarrow} \cdots \stackrel{\bar{\partial}}{\rightarrow} \Omega^{p, n}(M) \rightarrow 0
$$

and hence $\chi^{p}(M)=\sum_{q=0}^{n}(-1)^{q} h^{p, q}(M)$, where $h^{p, q}(M)$ are the corresponding Hodge numbers of $M$.

(2) For a general almost-complex manifold, $\bar{\partial}^{2}$ is not identically zero (it is a well-known fact that $\bar{\partial}^{2} \equiv 0$ is equivalent to the integrality of $J$ ). So we cannot define the Dolbeault complex (2.2). Therefore (2.1) may be taken as the Dolbeault-type complex in the almost-complex case.

(3) Using the general form of the Riemann-Roch-Hirzebruch theorem (first proved by Hirzebruch for projective manifolds [9], and in the general case by Atiyah and Singer [2]), we have

$$
\chi^{p}(M)=\left\langle\operatorname{ch}\left(\Lambda^{p} T^{*} M\right) \operatorname{td}(T M),[M]\right\rangle,
$$

where $\operatorname{ch}(\cdot)$ is the Chern character and $\operatorname{td}(\cdot)$ is the Todd class. $[M]$ is the fundamental class of $M$ induced from $J$ and $\langle\cdot, \cdot\rangle$ is the Kronecker pairing.

Now suppose $M$ admits a circle action $\left(S^{1}\right.$-action) preserving the almost-complex structure $J$. Then for any $g \in S^{1}$, we can define the equivariant index $\chi^{p}(g, M)$ (resp. equivariant Hirzebruch $\chi_{y}$-genus $\left.\chi_{y}(g, M):=\sum_{p=0}^{n} \chi^{p}(g, M) y^{p}\right)$ of the elliptic operators in (2.1) by choosing an invariant almost Hermitian metric under this circle action. Note that $\chi^{p}(g, M)$ is a finite Laurent series in $g$ as both $\operatorname{ker}\left(\bar{\partial}+\bar{\partial}^{*}\right)$ and $\operatorname{coker}\left(\bar{\partial}+\bar{\partial}^{*}\right)$ are finite-dimensional.

Moreover, we assume the fixed points of this action are nonempty and isolated, say $P_{1}, \cdots, P_{m}$. At each $P_{i}$, there are well-defined $n$ integer weights $k_{1}^{(i)}, \cdots, k_{n}^{(i)}$ (not necessarily distinct) induced from the isotropy representation of this $S^{1}$-action on $T_{p_{i}} M$. Note that these $k_{1}^{(i)}, \cdots, k_{n}^{(i)}$ are nonzero as the fixed points are isolated. We use $e_{i}\left(x_{1}, \cdots, x_{n}\right)(1 \leq i \leq n)$ to denote the $i$-th elementary symmetric polynomial of $x_{1}, \cdots, x_{n}$ and $e_{0}\left(x_{1}, \cdots, x_{n}\right):=1$. With this notation understood, the main observation in this paper is the following. 
Theorem 2.2. Let $\left(M^{2 n}, J\right)$ be a compact, connected, almost-complex manifold with a circle action preserving the almost-complex structure J. Suppose the fixed points of this action are nonempty and isolated. Let the notation be as above. Then for each $0 \leq p \leq n$, the expression

$$
\sum_{i=1}^{m} \frac{e_{p}\left(g^{k_{1}^{(i)}}, \cdots, g^{k_{n}^{(i)}}\right)}{\prod_{j=1}^{n}\left(1-g^{k_{j}^{(i)}}\right)}
$$

is a constant and equals $\chi^{p}(M)$. Here $g$ is an indeterminate.

Note that $\chi^{0}(M)$ is nothing else but the Todd genus of $M$. Hence combining with Fel'dman's observation (4], Theorems 1 and 2) mentioned in the Introduction, we have

Theorem 2.3. If $M$ is a symplectic manifold and the circle action is symplectic, then the expression

$$
\sum_{i=1}^{m} \frac{1}{\prod_{j=1}^{n}\left(1-g^{k_{j}^{(i)}}\right)}
$$

is either 0 , in which case the action is non-Hamiltonian, or 1 , in which case the action is Hamiltonian.

Our proof follows the exposition of Section 5.7 in 10 . Although the considerations of Sections 5.6 and 5.7 in [10] are for compact complex manifolds, we will see that, when replacing the Dolbeault complexes (2.2) by the elliptic complexes (2.1), the proof can also be applied to the situation of almost-complex manifolds with no more difficulties.

Proof of Theorem 2.2, Let $g \in S^{1}$ be a topological generator; i.e., the closure of $\left\{g^{r} \mid r \in \mathbb{Z}\right\}$ is the whole $S^{1}$. Then the fixed point set of the action $g$ is exactly $\left\{P_{1}, \cdots, P_{m}\right\}$. We note that the characteristic power series corresponding to the Hirzebruch $\chi_{y}$-genus is $x \frac{1+y e^{-x}}{1-e^{-x}}$. Then the Lefschetz fixed point formula of AtiyahBott-Segal-Singer ([2], p. 256) tells us that

$$
\chi_{y}(g, M)=\sum_{i=1}^{m} \prod_{j=1}^{n} \frac{1+y g^{k_{j}^{(i)}}}{1-g^{k_{j}^{(i)}}} .
$$

Note that the left hand side (LHS) of (2.3) is a finite Laurent series in $g$. Hence the only possible singularities are 0 and $\infty$, while

$$
\lim _{g \rightarrow 0} \prod_{j=1}^{n} \frac{1+y g^{k_{j}^{(i)}}}{1-g^{k_{j}^{(i)}}}=(-y)^{d_{i}}, \lim _{g \rightarrow \infty} \prod_{j=1}^{n} \frac{1+y g^{k_{j}^{(i)}}}{1-g^{k_{j}^{(i)}}}=(-y)^{n-d_{i}} .
$$

Here $d_{i}$ is the number of the negative integers in $k_{1}^{(i)}, \cdots, k_{n}^{(i)}$. By (2.4), the RHS of (2.3), and hence the LHS of (2.3), has well-defined limits at $g=0, \infty$. Therefore $\chi_{y}(g, M)$ must be constant in $g$ and

$$
\chi_{y}(g, M) \equiv \chi_{y}(i d, M)=\chi_{y}(M),
$$

which means that

$$
\chi_{y}(M)=\sum_{i=1}^{m} \prod_{j=1}^{n} \frac{1+y g^{k_{j}^{(i)}}}{1-g^{k_{j}^{(i)}}}
$$


holds for a dense subset of $S^{1}$ (the topological generators in $S^{1}$ are dense). So this must be an identity in the indeterminate $g$. Comparing the corresponding coefficients of (2.5), we have

$$
\chi^{p}(M)=\sum_{i=1}^{m} \frac{e_{p}\left(g^{k_{1}^{(i)}}, \cdots, g^{k_{n}^{(i)}}\right)}{\prod_{j=1}^{n}\left(1-g^{k_{j}^{(i)}}\right)}, 0 \leq p \leq n .
$$

This completes the proof of Theorem 2.2

Remark 2.4. (1) Let $N_{P}$ denote the number of fixed points of the circle action with exactly $p$ negative weights. From the proof we know that

$$
\begin{aligned}
\chi_{y}(M) & =\sum_{i=1}^{m}(-y)^{d_{i}}=\sum_{i=1}^{m}(-y)^{n-d_{i}} \\
& =\sum_{p=0}^{n} N_{p}(-y)^{p}=\sum_{p=0}^{n} N_{p}(-y)^{n-p}
\end{aligned}
$$

Hence

$$
\chi^{p}(M)=(-1)^{p} N_{p}=(-1)^{p} N_{n-p}=(-1)^{n} \chi^{n-p}(M) .
$$

(2) As pointed out in Section 5.7 of [10, this idea is essentially due to Lusztig [16], Kosniowski [1] and Atiyah-Hirzebruch [1]. The former two used it to derive the localization formula (2.6) for complex manifolds. Atiyah and Hirzebruch used this idea in [1] to get their famous vanishing theorem of $\hat{A}$-genus on spin manifolds.

(3) The localization formula (2.6) has been generalized to more general cases (cf. [8] and [14]) since its first appearance in [11] on complex manifolds. To the author's knowledge, in the case of almost-complex manifolds, the relations between $\chi^{p}(M)$ and the weights as in Theorem 2.2 have not been explicitly pointed out before. In fact, we will see in the next section that, with these relations set up, we can give many applications in related areas.

\section{Applications}

3.1. Since the appearance of [17, symplectic circle actions on six-dimensional symplectic manifolds have received a great deal of attention due to the rich structures and possibilities in this dimension. In [6, Godinho showed that certain symplectic circle actions on six-dimensional manifolds must be Hamiltonian. More precisely, let the circle act symplectically on a six-dimensional compact, connected, symplectic manifold with nonempty isolated fixed points whose isotropy weights are of the form $\left( \pm k_{1}, \pm k_{2}, \pm k_{3}\right)$ for fixed integers $k_{1} \geq k_{2} \geq k_{3} \geq 1$. Let $N_{0}$ and $N_{3}$ be the numbers of the fixed points with the weights $\left(k_{1}, k_{2}, k_{3}\right)$ and $\left(-k_{1},-k_{2},-k_{3}\right)$ respectively. Let $s_{1}, s_{2}, s_{3}, t_{1}, t_{2}$ and $t_{3}$ be the numbers of the weights $\left(-k_{1}, k_{2}, k_{3}\right),\left(k_{1},-k_{2}, k_{3}\right)$, $\left(k_{1}, k_{2},-k_{3}\right),\left(k_{1},-k_{2},-k_{3}\right),\left(-k_{1}, k_{2},-k_{3}\right)$ and $\left(-k_{1},-k_{2}, k_{3}\right)$ respectively. The following result is an extension of Godinho's results (cf. [6], Theorems 1.1, 1.2, 3.2 and 3.4).

Theorem 3.1. Let the circle act symplectically on a six-dimensional compact, connected, symplectic manifold with nonempty isolated fixed points and let the notation be as above. Then there are exactly two possibilities: 
(1) $N_{0}=N_{3}=s_{2}=s_{3}=t_{2}=t_{3}=0, s_{1}=t_{1} \geq 1$ and $k_{1}=k_{2}+k_{3}$, in which case the action is non-Hamiltonian;

(2) $N_{0}=N_{3}=1$ and

$$
\begin{aligned}
& \left(g^{k_{3}}+g^{k_{2}}+g^{k_{1}}\right)+\left(t_{1} g^{k_{2}+k_{3}}+t_{2} g^{k_{1}+k_{3}}+t_{3} g^{k_{1}+k_{2}}\right) \\
= & \left(s_{3} g^{k_{3}}+s_{2} g^{k_{2}}+s_{1} g^{k_{1}}\right)+\left(g^{k_{2}+k_{3}}+g^{k_{1}+k_{3}}+g^{k_{1}+k_{2}}\right),
\end{aligned}
$$

in which case the action is Hamiltonian. Here of course $g$ is an indeterminate.

Remark 3.2. When $k_{1} \neq k_{2}+k_{3}$, case (1) will lead to ([6], Theorems 1.1 and 3.2) and case (2) will lead to ([], Theorems 1.2 and 3.4).

Proof. Note that we have the following expression for the Todd class $\chi^{0}(M)$ :

$$
\begin{aligned}
& \chi^{0}(M)= \\
& \frac{N_{0}-\left(s_{1} g^{k_{1}}+s_{2} g^{k_{2}}+s_{3} g^{k_{3}}\right)+\left(t_{1} g^{k_{2}+k_{3}}+t_{2} g^{k_{1}+k_{3}}+t_{3} g^{k_{1}+k_{2}}\right)-N_{3} g^{k_{1}+k_{2}+k_{3}}}{\left(1-g^{k_{1}}\right)\left(1-g^{k_{2}}\right)\left(1-g^{k_{3}}\right)} .
\end{aligned}
$$

From Theorem 2.2 we have either

$$
N_{0}-\left(s_{1} g^{k_{1}}+s_{2} g^{k_{2}}+s_{3} g^{k_{3}}\right)+\left(t_{1} g^{k_{2}+k_{3}}+t_{2} g^{k_{1}+k_{3}}+t_{3} g^{k_{1}+k_{2}}\right)-N_{3} g^{k_{1}+k_{2}+k_{3}}=0,
$$

in which case the action is non-Hamiltonian, or

$$
\begin{aligned}
N_{0}- & \left(s_{1} g^{k_{1}}+s_{2} g^{k_{2}}+s_{3} g^{k_{3}}\right)+\left(t_{1} g^{k_{2}+k_{3}}+t_{2} g^{k_{1}+k_{3}}+t_{3} g^{k_{1}+k_{2}}\right)-N_{3} g^{k_{1}+k_{2}+k_{3}} \\
& =\left(1-g^{k_{1}}\right)\left(1-g^{k_{2}}\right)\left(1-g^{k_{3}}\right),
\end{aligned}
$$

in which case the action is Hamiltonian.

We can also reproduce Tolman-Weitsman's following result ([19], Theorem 1).

Theorem 3.3 (Tolman-Weitsman). Let $M^{2 n}$ be a compact, connected symplectic manifold, equipped with a semifree symplectic circle action with nonempty isolated fixed points. Then this circle action must be Hamiltonian.

Proof. Since the action is semifree, all the integer weights $k_{j}^{(i)}$ are \pm 1 . We still use $N_{p}$ to denote the number of fixed points with exactly $p$ negative weights as in Section 2. Hence

$$
\chi^{0}(M)=\frac{\sum_{p=0}^{n} N_{p}(-g)^{p}}{(1-g)^{n}} .
$$

By assumption, the fixed points are nonempty; hence at least one of $N_{0}, \cdots, N_{n}$ is nonzero, which means that $\chi^{0}(M) \neq 0$. According to Theorem $2.2, \chi^{0}(M)=1$ and the action is Hamiltonian. Moreover, $N_{p}=\left(\begin{array}{l}n \\ p\end{array}\right), 0 \leq p \leq n$. (Compare to Lemma 3.1 in [19.) 
Remark 3.4. This result was also re-proved by Fel'dman (4], Corollary 1), whose main tools are the Conner-Floyd equations for the Todd class.

3.2. We still use the notation in Section 2. In this subsection we will prove the following result.

Theorem 3.5. Suppose the almost-complex manifold $\left(M^{2 n}, J\right)$ admits a circle action preserving the almost-complex structure $J$ and having isolated fixed points. Then for any $k \in \mathbb{Z}-\{0\}$,

$$
\sharp\left\{k_{j}^{(i)}=k \mid 1 \leq i \leq m, 1 \leq j \leq n\right\}=\sharp\left\{k_{j}^{(i)}=-k \mid 1 \leq i \leq m, 1 \leq j \leq n\right\} .
$$

Here $\sharp$ denotes the cardinality of a set.

The following corollary is a generalization of ([18, Theorem 2) from the case of symplectic circle actions on symplectic manifolds to that of circle actions on almost-complex manifolds.

Corollary 3.6. Suppose the almost-complex manifold $\left(M^{2 n}, J\right)$ admit a circle action preserving the almost-complex structure $J$ and having isolated fixed points. Then

$$
\sum_{i=1}^{m} \sum_{j=1}^{n} k_{j}^{(i)}=0
$$

In order to derive Theorem 3.5, we need the following lemma.

Lemma 3.7. $\forall 0 \leq k \leq n$, we have

$$
e_{k}\left(x_{1}+1, x_{2}+1, \cdots, x_{n}+1\right)=\sum_{i=0}^{k}\left(\begin{array}{c}
n-i \\
n-k
\end{array}\right) e_{i}\left(x_{1}, x_{2}, \cdots, x_{n}\right) \text {. }
$$

Proof.

$$
\begin{aligned}
e_{k}\left(x_{1}+1, x_{2}+1, \cdots, x_{n}+1\right) & =\sum_{1 \leq i_{1}<\cdots<i_{k} \leq n}\left(x_{i_{1}}+1\right)\left(x_{i_{2}}+1\right) \cdots\left(x_{i_{k}}+1\right) \\
& :=\sum_{i=0}^{k} a_{i} \cdot e_{i}\left(x_{1}, x_{2}, \cdots, x_{n}\right) .
\end{aligned}
$$

It suffices to determine the coefficients $a_{i}$. Note that there are $\left(\begin{array}{l}n \\ k\end{array}\right)\left(\begin{array}{l}k \\ i\end{array}\right)$ monomials of degree $i$ in the expression $\sum_{1 \leq i_{1}<\cdots<i_{k} \leq n}\left(x_{i_{1}}+1\right)\left(x_{i_{2}}+1\right) \cdots\left(x_{i_{k}}+1\right)$. Hence,

$$
a_{i}=\frac{\left(\begin{array}{l}
n \\
k
\end{array}\right)\left(\begin{array}{l}
k \\
i
\end{array}\right)}{\left(\begin{array}{l}
n \\
i
\end{array}\right)}=\left(\begin{array}{c}
n-i \\
n-k
\end{array}\right)
$$

as $e_{i}\left(x_{1}, \cdots, x_{n}\right)$ has $\left(\begin{array}{c}n \\ i\end{array}\right)$ monomials. 
Proof of Theorem 3.5 .

$$
\begin{aligned}
\sum_{i=1}^{m} \sum_{j=1}^{n} \frac{1}{1-g^{k_{j}^{(i)}}} & =\sum_{i=1}^{m} \frac{e_{n-1}\left(1-g^{k_{1}^{(i)}}, \cdots, 1-g^{k_{n}^{(i)}}\right)}{\prod_{j=1}^{n}\left(1-g^{k_{j}^{(i)}}\right)} \\
& =\sum_{l=0}^{n-1}(n-l)(-1)^{l} \sum_{i=1}^{m} \frac{e_{l}\left(g^{k_{1}^{(i)}}, \cdots, g^{k_{n}^{(i)}}\right)}{\prod_{j=1}^{n}\left(1-g^{k_{j}^{(i)}}\right)} \text { (by Lemma 3.7) } \\
& =\sum_{l=0}^{n-1}(n-l)(-1)^{l} \chi^{l}(M) \\
& =\sum_{l=0}^{n-1}(n-l) N_{l} \\
& =\frac{n}{2} \sum_{l=0}^{n} N_{l}\left(\text { by } N_{l}=N_{n-l}\right) \\
& =\frac{m n}{2} .
\end{aligned}
$$

Note that for any $k \in \mathbb{Z}-\{0\}, \frac{1}{1-g^{k}}+\frac{1}{1-g^{-k}}=1$. So what we have showed implies that, for any $k \in \mathbb{Z}-\{0\}$,

$$
\sharp\left\{k_{j}^{(i)}=k \mid 1 \leq i \leq m, 1 \leq j \leq n\right\}=\sharp\left\{k_{j}^{(i)}=-k \mid 1 \leq i \leq m, 1 \leq j \leq n\right\} .
$$

\section{ACKNOWLEDGEMENT}

The author thanks the referee for a very careful reading of the earlier version of this paper and for many fruitful comments and suggestions which improved the quality of the paper.

\section{REFERENCES}

[1] M.F. Atiyah, F. Hirzebruch: Spin-Manifolds and Group Actions, Essays on Topology and Related Topics, Mémoires dédiés à Georges de Rham, A. Haefliger and R. Narasimhan, eds., Springer-Verlag, New York-Berlin, 1970, pp. 18-28. MR.0278334 (43:4064)

[2] M.F. Atiyah, I.M. Singer: The index theory of elliptic operators: III, Ann. of Math. (2) 87 (1968), 546-604. MR0236952 (38:5245)

[3] R. Bott, C. Taubes: On the rigidity theorems of Witten, J. Amer. Math. Soc. 2 (1989), 137-186. MR 954493 (89k:58270)

[4] K. È. Fel'dman: Hirzebruch genus of a manifold supporting a Hamiltonian circle action, Russian Math. Surveys 56 (2001), 978-979. MR.1892568 (2003a:57062)

[5] T. Frankel: Fixed points and torsion on Kähler manifolds, Ann. of Math. (2) 70 (1959), 1-8. MR0131883 (24:A1730)

[6] L. Godinho: On certain symplectic circle actions, J. Symplectic Geom. 3 (2005), 357-383. MR2198781 (2006k:57074)

[7] P. Griffiths, J. Harris: Principles of algebraic geometry, Pure and Applied Mathematics, Wiley, New York, 1978. MR.507725 (80b:14001)

[8] A. Hattori, H. Taniguchi: Smooth $S^{1}$-action and bordism, J. Math. Soc. Japan. 24 (1972), 701-731. MR0309134 (46:8245)

[9] F. Hirzebruch: Topological methods in algebraic geometry, 3rd edition, Springer, Berlin, 1966. MR0202713 (34:2573)

[10] F. Hirzebruch, T. Berger, R. Jung: Manifolds and modular forms, Aspects of Mathematics, E20, Friedr. Vieweg and Sohn, Braunschweig, 1992. MR.1189136 (94d:57001)

[11] C. Kosniowski: Applications of the holomorphic Lefschetz formula, Bull. London Math. Soc. 2 (1970), 43-48. MR0261636 (41:6249) 
[12] C. Kosniowski: Holomorphic vector fields with simple isolated zeros, Math. Ann. 208 (1974), 171-173. MR0341501 (49:6252)

[13] C. Kosniowski: Some formulae and conjectures associated with circle actions, Topology Symposium, Siegen, 1979 (Proc. Sympos., Univ. Siegen, Siegen, 1979), Lecture Notes in Math., 788, Springer, Berlin, 1980, pp. 331-339. MR585666 (81k:57036)

[14] C. Kosniowski, M. Yahia: Unitary bordism of circle actions, Proc. Edinb. Math. Soc. 26 (1983), 97-105. MR695647 (84e:57032)

[15] H.B. Lawson, M.L. Michelson: Spin geometry, Princeton Univ. Press, Princeton, New Jersey, 1989. MR.1031992 (91g:53001)

[16] G. Lusztig: Remarks on the holomorphic Lefschetz numbers, pp. 193-204 in: Analyse globale (Sém. Math. Supérieures, No. 42, 1969). Presses Univ. Montréal, Montreal, Que., 1971. MR0380821 (52:1718)

[17] D. McDuff: The moment map for circle actions on symplectic manifolds, J. Geom. Phys. 5 (1988), 149-160. MR1029424 (91c:58042)

[18] A. Pelayo, S. Tolman: Fixed points of symplectic periodic flows, Ergodic Theory and Dynamical Systems 31 (2011), 1237-1247.

[19] S. Tolman, J. Weitsman: On semifree symplectic circle actions with isolated fixed points, Topology 39 (2000), 299-309. MR.1722020(2000k:53074)

Department of Mathematics, Tongui University, Shanghai 200092, People's Republic OF CHINA

E-mail address: pingli@tongji.edu.cn 